\title{
Long-term rivaroxaban for the treatment of acute venous thromboembolism in patients with active cancer in a prospective multicenter trial
}

\author{
Ho-Young Yhim ${ }^{1,2}$, Won-Il Choi ${ }^{3}$, Sung-Hyun Kim ${ }^{4}$, Seung-Hyun Nam ${ }^{5}$, Kyoung Ha Kim ${ }^{6}$, \\ Yeung-Chul Mun ${ }^{7}$, Doyeun $\mathrm{Oh}^{8}$, Hun-Gyu Hwang ${ }^{9}$, Keun-Wook Lee ${ }^{10}$, Eun-Kee Song ${ }^{1,2}$, \\ Yong Shik Kwon ${ }^{3}$, and Soo-Mee Bang ${ }^{10}$
}

\begin{abstract}
${ }^{1}$ Department of Internal Medicine, Chonbuk National University Medical School, Jeonju; ${ }^{2}$ Research Institute of Clinical Medicine of Chonbuk National UniversityBiomedical Research Institute of Chonbuk National University Hospital, Jeonju; ${ }^{3}$ Department of Internal Medicine, Keimyung University Dongsan Medical Center Daegu; ${ }^{4}$ Department of Internal Medicine, Dong-A University College of Medicine, Busan; ${ }^{5}$ Department of Internal Medicine, Veterans Health Service Medical Center, Seoul; ${ }^{6}$ Department of Internal Medicine, Soonchunhyang University Seoul Hospital, Seoul; ${ }^{7}$ Department of Internal Medicine, Ewha Womans University School of Medicine, Seoul; ${ }^{8}$ Department of Internal Medicine, CHA University School of Medicine, Seongnam; ${ }^{9}$ Department of Internal Medicine, Soonchunhyang University Gumi Hospital, Gumi;

${ }^{10}$ Department of Internal Medicine, Seoul National University Bundang Hospital, Seongnam, Korea
\end{abstract}

Received: March 9, 2018 Revised : April 10, 2018 Accepted: April 11, 2018

\section{Correspondence to}

Soo-Mee Bang, M.D.

Department of Internal Medicine, Seoul National University Bundang Hospital, 82 Gumi-ro 173beon-gil, Bundang-gu, Seongnam 13620,

Korea

Tel: +82-31-787-7039

Fax: $+82-31-787-4098$

E-mail: smbang7@snu.ac.kr
Background/Aims: Limited data are available regarding the efficacy of rivaroxaban for the treatment of cancer-associated venous thromboembolism (VTE). The aim of this study was to evaluate the effectiveness and safety of rivaroxaban for the treatment of VTE in active cancer patients.

Methods: In this prospective, multicenter, open-label trial (NCTo1989845), we enrolled patients with active cancer and objectively diagnosed lower-extremity deep vein thrombosis, pulmonary embolism (PE), or both from November 2013 to June 2016. Active cancer was defined as a histologically confirmed malignancy, which was diagnosed or treated within the previous 6 months, or as a recurrent/ metastatic cancer. Patients received oral rivaroxaban $15 \mathrm{mg}$ twice daily for first 3 weeks, followed by $20 \mathrm{mg}$ once daily for 6 months. The primary outcome was the symptomatic recurrent VTE and the secondary outcomes included any recurrent VTE, major or clinically relevant non-major (CRNM) bleeding events, and overall mortality. All study outcomes were validated by blinded central adjudication.

Results: Of 124 patients enrolled, 110 (88.7\%) had solid cancer, 93 (75.0\%) had metastatic disease, and $110(88.7 \%)$ were receiving chemotherapy or radiotherapy. During the 6-month study period, seven patients experienced symptomatic recurrent VTE (cumulative incidence, 5.9\%), and two patients experienced incidental recurrent PE (cumulative incidence of any recurrent VTE, 7.6\%). Major bleeding events occurred in six patients (cumulative incidence, 5.3\%) and CRNM bleeding events in 11 patients (cumulative incidence, 10.2\%). Twenty-eight patients (overall mortality, 24.0\%) died.

Conclusions: Rivaroxaban is effective and safe for the treatment of VTE in patients with active cancer.

Keywords: Neoplasms; Recurrence; Rivaroxaban; Venous thromboembolism; Therapeutics 


\section{INTRODUCTION}

Venous thromboembolism (VTE), which typically includes lower-extremity deep vein thrombosis (DVT) and pulmonary embolism (PE), is a common complication that can be associated with considerable morbidity and mortality in cancer patients [1]. Based on the results of large randomized trials in patients with cancer $[2,3]$, the current international practice guidelines recommend the long-term use of low-molecular-weight heparin (LMWH) for the treatment of VTE in cancer patients [4-7].

The recent introduction of direct oral anticoagulants (DOACs) that directly inhibit clotting factor Xa or thrombin is rapidly replacing the use of LMWH and vitamin K antagonists in the treatment of VTE [4]. DOACs are attractive drugs in patients with VTE because they can be administered orally with fixed doses, they have few drug and food interactions, and they do not require routine laboratory monitoring [8]. Despite the practical advantages of DOACs, these agents have not been recommended for treatment of cancer-associated VTE in the international guidelines due to insufficient evidence [4-7]. Although the pivotal phase III trials testing DOACs included cancer patients, the results of these trials [9-13] and subsequent subgroup analysis [14-17] have been underpowered by the small number of cancer patients and the heterogeneity in definition of cancer for the trials [18]. Indeed, the cancer patients included in the pivotal trials usually had completed the cancer treatment before enrollment or had a previous history of cancer and eventually they were not a true representative of active cancer patients [19]. Thus, there is considerable debate regarding the use of DOACs in the treatment of VTE in patients with active cancer. Therefore, we conducted this study (KVTE-1301) to evaluate the effectiveness and safety of long-term rivaroxaban treatment in patients with active cancer and VTE.

\section{METHODS}

\section{Study population and eligibility criteria}

The present prospective, multicenter, open-label study was designed to investigate the effectiveness and safety of oral rivaroxaban treatment in consecutive patients with active cancer and objectively diagnosed lower-ex- tremity DVT, PE, or both, who had a life expectancy of $\geq$ 3 months. The study was conducted in nine institutions of the Thrombosis Working Party of the Korean Society of Hematology and Venous Thrombosis Division of the Korean Society of Thrombosis and Hemostasis. Active cancer was defined as a histologically confirmed solid cancer or hematologic malignancy (except for basal cell or squamous cell carcinoma of the skin), which were diagnosed or treated within the previous 6 months, or as a recurrent/ metastatic cancer. $\mathrm{PE}$ was diagnosed based on the presence of non-enhancing filling defects in at least one subsegmental pulmonary artery on computed tomography (CT) pulmonary angiography, or high probability mismatched perfusion defect on ventilation/perfusion lung scan. DVT was diagnosed based on the non-compressibility on ultrasonography, or intraluminal filling defects in lower-extremity deep vein on CT venography. Incidental PE, which was defined when $\mathrm{PE}$ was detected by imaging studies that were performed for reasons other than suspected PE, was considered if the equivalent diagnostic criteria for symptomatic PE were met.

Patients were excluded if they had isolated asymptomatic distal DVT, splanchnic vein thrombosis, or catheter-associated thrombosis; an Eastern Cooperative Oncology Group (ECOG) performance status 3 or 4 ; a creatinine clearance of $<30 \mathrm{~mL} / \mathrm{min} / 1.73 \mathrm{~m}^{2}$; history of total gastrectomy; confirmation of VTE or therapeutic anticoagulation for $>120$ hours prior to the first dose of rivaroxaban after enrollment; cardiogenic shock; treatment of qualifying VTE with thrombolysis, caval filter, or thrombectomy; uncontrolled brain metastasis; cancer treatment with bevacizumab or other drugs with potent anti-angiogenic property; active bleeding or history of bleeding within 4 weeks before enrollment; a high risk of serious bleeding; a platelet count $<75,000 / \mu \mathrm{L}$; pregnancy or breast feeding.

\section{Study treatment}

Rivaroxaban should be administered within 120 hours after confirmation of the VTE or therapeutic anticoagulation initiation. Patients were treated with oral rivaroxaban $15 \mathrm{mg}$ twice daily for the first 3 weeks, followed by $20 \mathrm{mg}$ once daily for 6 months. Rivaroxaban was temporarily withheld if patients had a platelet count of < 50,000/ $\mu \mathrm{L}$, bleeding events, or invasive procedures. Dose reduction was not permitted, unless patients experi- 
enced major bleeding events.

\section{Study outcomes and follow-up}

The primary outcome of this study was to assess the symptomatic recurrent VTE during the 6-month period, which included fatal or symptomatic non-fatal PE or DVT. Fatal PE was defined when patients were confirmed by objective diagnostic testing or autopsy, or when a specific cause of death could not be documented and PE could not be ruled out. Recurrent non-fatal PE was defined as a new intraluminal filling defect on CT pulmonary angiography, or as the presence of new or clearly enlarged area of mismatched perfusion defect on ventilation/perfusion lung scan. Recurrent DVT was determined if new thrombus was clearly identified by non-compressibility on ultrasonography or new intraluminal filling defect on venography.

The secondary outcomes included any (symptomatic or incidental) recurrent VTE, bleeding events (major bleeding or clinically relevant non-major [CRNM] bleeding), overall mortality, and validation of the Ottawa score [20]. The severity of bleeding events was classified based on the criteria of the International Society on Thrombosis and Haemostasis [21]. All recurrent VTE episodes, bleeding events, and deaths were reviewed in the independent central adjudication committee. They confirmed the presence of recurrent VTE, graded bleeding event severities, and determined the cause of death.

Patients had regular follow-up visit on days 7, 21 and then every 28 days until day 180, consent withdrawal, lost to follow-up or death. During every visit, symptoms and signs of recurrent VTE and bleeding events were thoroughly investigated by treating physicians. If patients developed symptoms and signs of recurrent VTE, appropriate imaging methods were implemented to objectively diagnose VTE. Telephone contacts were made in case patients did not visit the hospital on the scheduled day.

\section{Statistical analysis}

Recurrent VTE rates for active cancer patients treated with LMWH were reported previously to be $9 \%$ [2]. Sample size was calculated using one-sample proportion test to be sufficient to reject if expected proportion of recurrence was higher than $9 \%$ with a two-sided $95 \%$ confidence interval (CI) of 0.107. Planned accrual was for
110 patients. Assuming a 15\% dropout rate, the total accrual needed to be 127 patients. The recurrent VTE and bleeding events were calculated using a cumulative incidence method that incorporated death from any cause as competing risk [22]. Mortality was estimated using the Kaplan-Meier method. All analyses were conducted using SAS Enterprise Guide version 6.4 (SAS Institute Inc., Cary, NC, USA).

\section{Study oversight}

The study was conducted in accordance with the Good Clinical Practice of the International Conference on Harmonization Guidelines and the Declaration of Helsinki. The investigators were responsible for the study design and Bayer Korea provided an independent research fund to conduct the study. The study protocol was approved by the Institutional Review Board (B1308/216-005) of each participating institution and registered at www.clinicaltrials.gov (NCTo1989845). All patients provided written informed consent.

\section{RESULTS}

\section{Patient cohort and anticoagulation treatment}

A total of 127 patients were screened for this study from November 2013 to June 2016. Three patients were excluded because they did not meet the eligibility criteria: one patient did not have qualifying thrombotic episodes, one patient had a creatinine clearance of $<30 \mathrm{~mL} /$ $\min / 1.73 \mathrm{~m}^{2}$, and one patient had ECOG performance status 3. Thus, 124 patients were enrolled and the data for these were analyzed (Fig. 1).

Baseline clinical and thrombotic event characteristics of the 124 patients are described in Table 1. Of these, $88.7 \%$ of patients had solid cancers, $75.0 \%$ had metastatic disease, and $88.7 \%$ were receiving chemotherapy or radiotherapy. The most common primary tumor sites were lung, colorectum, pancreaticobiliary tract, and stomach. The qualifying VTE event was $\mathrm{PE} \pm$ DVT in $82.3 \%$ of the patients and presented as symptomatic in $62.9 \%$ of the patients.

Rivaroxaban was administered for a median duration of 171 days (range, 1 to 208) and temporarily interrupted in 32 patients (25.8\%) for a median of 8 days (range, 1 to 37 ). 


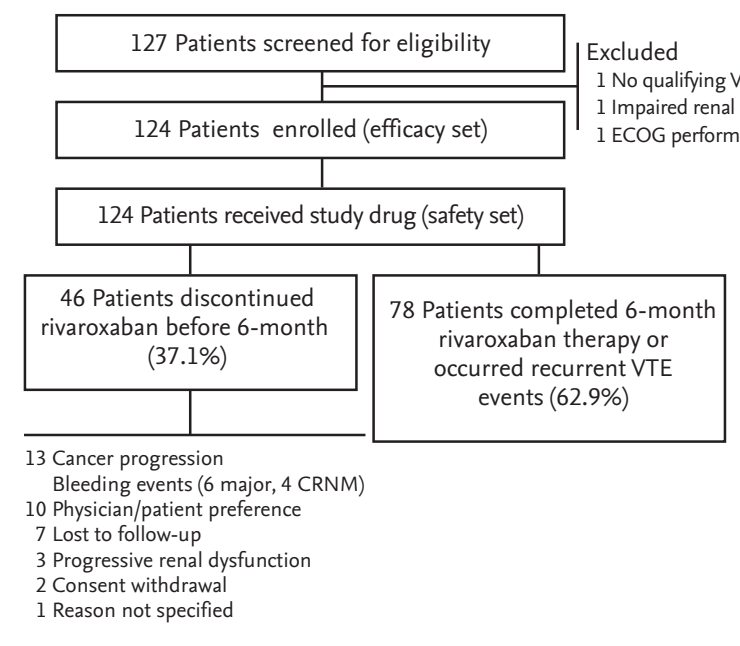

Figure 1. Flow-chart of the study. VTE, venous thromboembolism; ECOG, Eastern Cooperative Oncology Group; CRNM, clinically relevant non-major.

\section{Recurrent VTE episodes}

Seven patients experienced symptomatic recurrent VTE episodes: fatal PE in four patients and symptomatic non-fatal PE in three patients. The cumulative incidence of symptomatic recurrent VTE was 5.9\% (95\% CI, 2.6 to 11.1) at 6 months (Fig. 2A). Two patients experienced incidental recurrent $\mathrm{PE}$. Thus, the cumulative incidence of any recurrent VTE was 7.6\% (95\% CI, 3.7 to 13.2) (Fig. 2B). Comparisons of cumulative risk of recurrent VTE according to the symptom status at the time of qualifying VTE diagnosis showed no significant differences between symptomatic and incidental VTE events (symptomatic recurrence, $8.1 \%$ vs. $2.2 \%$, respectively, $p$ $=0.184$; any recurrence, $9.4 \%$ vs. $4.5 \%$, respectively, $p=$ 0.300). The Ottawa score did not predict the recurrence of symptomatic or any VTE events (Fig. 3). Clinical and thrombotic characteristics of patients with symptomatic recurrent VTE were summarized in Table 2.

\section{Bleeding events and mortality}

Six and 11 patients experienced major and CRNM bleeding events, respectively. The most common site of major bleeding was gastrointestinal in four patients (66.7\%), whereas one patient experience major bleeding in the lung and one showed intracranial bleeding. Unresected primary tumor in the gastrointestinal tract was the source of major bleeding in two patients (33.3\%). The cumulative incidence of major and CRNM bleeding events was 5.3\% (95\% CI, 2.1 to 10.5) and 10.2\% (95\% CI, 5.3 to 16.9), respectively (Fig. 4). All six patients experienced major bleeding events didn't resume rivaroxaban treatment.

Overall, 28 patients died; the cause of death was cancer progression in 17 patients (60.7\%), fatal $\mathrm{PE}$ in four patients (14.3\%), infection in four patients (14.3\%), and fatal bleeding events in three patients (10.7\%). The overall mortality was $24.0 \%$ (95\% CI, 15.8 to 31.4 ) at 6 months (Fig. 5). The mortality rate of patients with symptomatic VTE events was not significantly different compared to that of patients with incidental VTE events (23.4\% vs. $24.7 \%$, respectively; $p=0.998$ ).

\section{DISCUSSION}

Our study provides several valuable insights into the incorporation of DOACs as a therapeutic option for cancer-associated VTE. Our results show that rivaroxaban is effective in patients with active cancer and lower-extremity DVT, PE, or both. In 124 patients, the majority of whom had metastatic disease and were under anti-cancer treatments, we observed a cumulative risk of symptomatic recurrence of $5.9 \%$ and a rate of any recurrence of $7.6 \%$. The risk of recurrence observed in this study was promising, as compared with approximately $7 \%$ to $9 \%$ observed in active cancer patients treated with LMWH in the CLOT and CATCH trials (Table 3) [2,3]. In contrast, the results of meta-analysis [23] and subgroup analysis [14-17] regarding cancer patients enrolled in the pivotal DOAC trials showed that the recurrent VTE rates were approximately $3 \%$ to $5 \%$. Although the recurrence rates of cancer patients in the DOAC trials were lower than those observed in the LMWH trials, it is difficult to be generalized in favor of the use of DOACs for treatment of cancer-associated VTE due to the different definition of cancer patients between the DOAC and LMWH trials. Both CLOT and CATCH trials included patients with recently diagnosed or treated cancer, or with recurrent/metastatic cancer in palliative setting $[2,3]$. In contrast, a substantial number of cancer patients in the DOAC trials just had a history of cancer at study entry. Trials for apixaban [12] and edoxaban [13], in particular, excluded patients requiring long-term anticoagulation therapy, which indicated patients with recurrent/meta- 
Table 1. Baseline clinical and thrombotic event characteristics

\begin{tabular}{lc}
\hline Characteristic & Patients $(\mathrm{n}=124)$ \\
\hline Age, yr & $67(28-85)$ \\
Sex & \\
Male & $69(55.6)$ \\
Female & $55(44.4)$
\end{tabular}

Primary site of cancer

\begin{tabular}{|lc|}
\hline Lung & $30(24.2)$ \\
\hline Colorectum & $24(19.4)$ \\
\hline Pancreaticobiliary tract & $16(12.9)$ \\
\hline Stomach & $16(12.9)$ \\
\hline Hematologic malignancy & $14(11.3)$ \\
\hline Gynecologic system & $9(7.3)$ \\
\hline Breast & $7(5.6)$ \\
\hline Genitourinary tract & $4(3.2)$ \\
\hline Other $^{\mathrm{a}}$ & $4(3.2)$ \\
\hline
\end{tabular}

Extent of disease ${ }^{\mathrm{b}}$

$\begin{array}{lc}\text { Localized or locoregional disease } & 17(13.7) \\ \text { Metastatic disease } & 93(75.0) \\ \text { Cancer treatment } & 110(88.7) \\ \text { Yes } & 109(87.9) \\ \text { Systemic anti-cancer treatments } & \\ \text { Radiotherapy }^{\mathrm{d}} & 6(4.8) \\ \text { Recent major surgery within } 6 \text { months }^{\text {ECOG performance status }} & 23(18.5)\end{array}$

$\begin{array}{ll}\text { O or } 1 & 97(78.2) \\ 2 & 27(21.8)\end{array}$

2

$27(21.8)$

The Ottawa score ${ }^{\mathrm{e}}$

$\begin{array}{lc}\text { Low risk }(\leq 0) & 53(42.7) \\ \text { High risk }(>0) & 71(57.3) \\ \text { Previous history of VTE } & 6(4.8) \\ \text { Body weight, kg } & 61.2 \pm 10.9 \\ \text { Body mass index } & 23.9 \pm 3.9 \\ \text { Creatinine clearance }{ }^{\mathrm{f}}, \mathrm{mL} / \mathrm{min} / 1.73 \mathrm{~m}^{2} & \\ \geq 30.0 \text { and }<50.0 & 87(14.5) \\ \geq 50.0 \text { and }<80.0 & 67(54.0) \\ \geq 80.0 & 39(31.5) \\ \text { Qualifying VTE events } & \end{array}$

Table 1. Continued

\begin{tabular}{lc}
\hline Characteristic & Patients $(\mathrm{n}=124)$ \\
\hline PE with or without lower-extremity DVT & $102(82.3)$ \\
\hline Lower-extremity DVT alone & $22(17.7)$ \\
\hline Symptom status of qualifying VTE events & \\
\hline Symptomatic & $78(62.9)$ \\
\hline Incidental & $46(37.1)$ \\
\hline Largest vessel involved & $102(100.0)$ \\
\hline Pulmonary artery & $16(15.7)$ \\
\hline Main & $41(40.2)$ \\
\hline Lobar & $43(42.2)$ \\
\hline Segmental & $2(2.0)$ \\
\hline Subsegmental & $46(100.0)$ \\
\hline Lower extremity deep vein & $41(89.1)$ \\
\hline Proximal vein & $5(10.9)$ \\
\hline Distal veing &
\end{tabular}

Values are presented as mean (range), number(\%), or mean \pm SD. ECOG, Eastern Cooperative Oncology Group; VTE, venous thromboembolism; DVT, deep vein thrombosis.

${ }^{a}$ Other cancers include nasopharyngeal cancer $(n=2)$, head and neck squamous cell carcinoma $(n=1)$, and metastasis of unknown primary site $(\mathrm{n}=1)$.

${ }^{b}$ Patients with hematologic malignancy were not included.

${ }^{\mathrm{c}}$ Systemic anti-cancer treatments included cytotoxic, hormonal, and targeted therapy.

${ }^{\mathrm{d}}$ Five patients were receiving concurrent chemoradiotherapy.

'The Ottawa score that assigned a score of -2 for stage I cancer, -1 for breast cancer, and +1 for lung cancer, female sex, and previous history of VTE categorized low risk group for recurrent VTE when the sum of score was $\leq \mathrm{o}$ and high risk group if the sum of score was $>0$.

${ }^{\mathrm{f}}$ Creatinine clearance was calculated by the Cockcroft and Gault formulas.

g One patient who had been receiving adjuvant chemotherapy for resected lung cancer had symptomatic isolated distal DVT, and another four patients with distal DVT had concurrent PE.

static cancer were not eligible. Therefore, considering that no studies have specifically addressed the treatment of VTE using DOACs in patients with active cancer, it is noteworthy that our study tested rivaroxaban using the same definition of active cancer as in the CLOT and CATCH trials.

Because the Korean population was generally consid- 


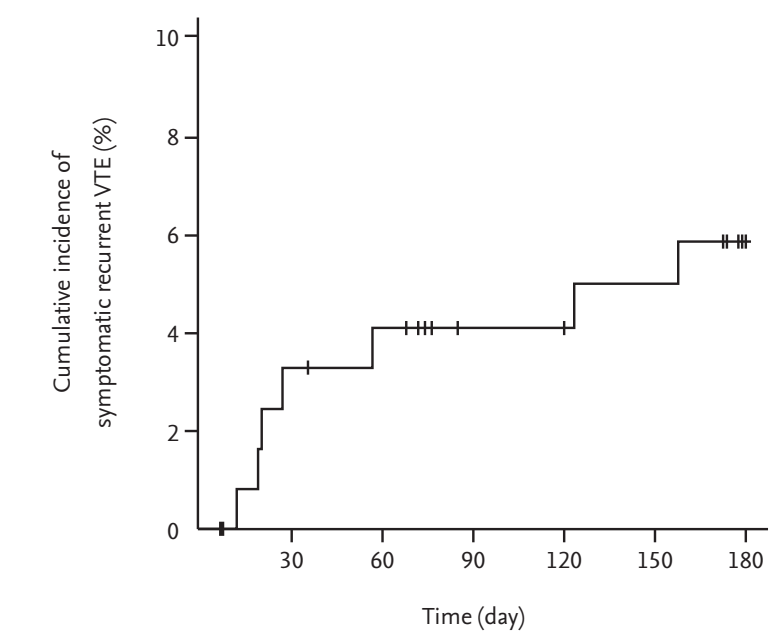

A

No. at risk

124

$116 \quad 108$

102

$98 \quad 89$

80

B

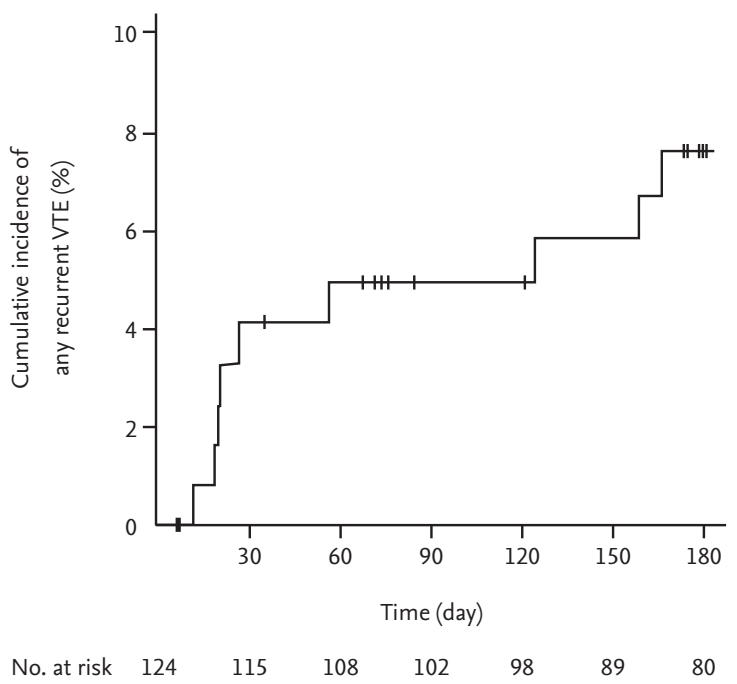

Figure 2. Cumulative incidence of (A) symptomatic and (B) any recurrent venous thromboembolism (VTE).

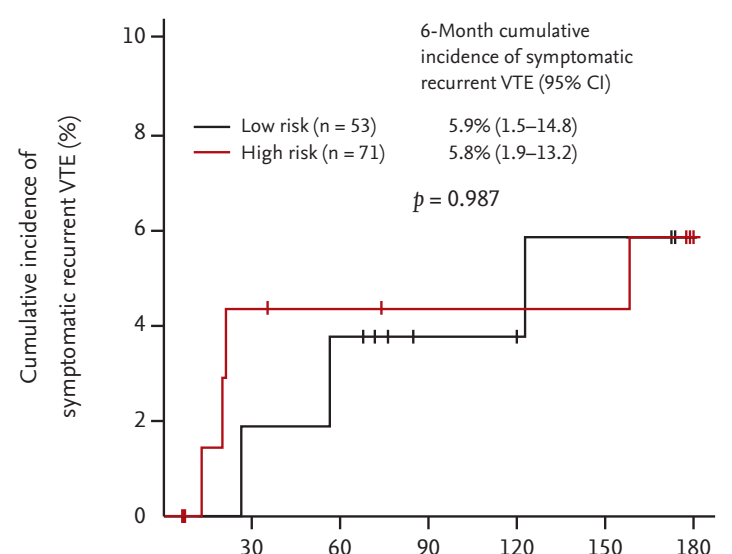

No. at risk

Low risk

High risk

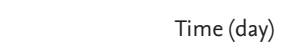

Figure 3. Cumulative incidence of (A) symptomatic and (B) any recurrent venous thromboembolism (VTE) according to the risk stratification by Ottawa score. CI, confidence interval.

ered to have lower risk of VTE [24] even in surgical oncologic patients [25], as compared with Caucasian populations, our data should be interpreted with caution. However, there have been recent data suggesting that the tumor activity of advanced stage cancer can overcome the ethnic protective effect in the Korean population regarding thrombosis and recurrent thrombosis $[26,27]$. In our study, the mean age was 67 years (for CLOT, 62 years; for CATCH, 60 years), $75 \%$ of the patients had metastatic disease (for CLOT, 67\%; for CATCH, 55\%),

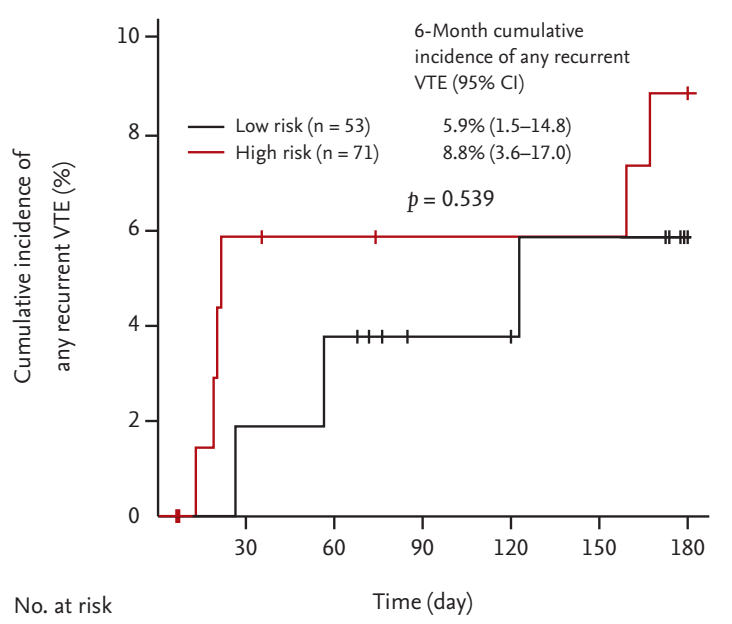

$\begin{array}{llllllll}\text { B Low risk } & 53 & 51 & 48 & 43 & 40 & 36 & 31 \\ \text { High risk } & 71 & 64 & 60 & 59 & 58 & 53 & 49\end{array}$ 
Table 2. Clinical and thrombotic characteristics of patients with symptomatic recurrent VTE $(\mathbf{n}=7)$

\begin{tabular}{|c|c|c|c|c|c|c|c|}
\hline Characteristic & Case 1 & Case 2 & Case 3 & Case 4 & Case 5 & Case 6 & Case 7 \\
\hline Age & 54 & 65 & 81 & 71 & 64 & 75 & 81 \\
\hline Sex & Male & Male & Male & Female & Male & Male & Male \\
\hline Primary site of cancer & Pancreas & Pancreas & Lung & Lung & Lung & Lung & Colon \\
\hline Extent of disease & Metastatic & Metastatic & Metastatic & Metastatic & Metastatic & Metastatic & Metastatic \\
\hline Ottawa score & Low risk & Low risk & High risk & High risk & High risk & High risk & Low risk \\
\hline $\begin{array}{l}\text { Qualifying VTE } \\
\text { events and } \\
\text { involved vessels }\end{array}$ & $\begin{array}{l}\text { Rt. lobar PE and } \\
\text { Rt. proximal } \\
\text { DVT }\end{array}$ & $\begin{array}{l}\text { Both main PE } \\
\text { and Lt. proxi- } \\
\text { mal DVT }\end{array}$ & Rt. lobar PE & $\begin{array}{l}\text { Lt. lower } \\
\text { segmental } \\
\text { PE }\end{array}$ & $\begin{array}{l}\text { Both main } \\
\text { PE }\end{array}$ & Rt. lobar PE & $\begin{array}{l}\text { Rt. proximal } \\
\text { DVT }\end{array}$ \\
\hline $\begin{array}{l}\text { Symptom status of } \\
\text { qualifying VTE }\end{array}$ & Symptomatic & Incidental & Symptomatic & Symptomatic & Symptomatic & Symptomatic & Symptomatic \\
\hline $\begin{array}{l}\text { Fatal or non-fatal } \\
\text { recurrent PE events }\end{array}$ & $\begin{array}{l}\text { Non-fatal both } \\
\text { lower } \\
\text { segmental PE }\end{array}$ & $\begin{array}{l}\text { Non-fatal both } \\
\text { main and } \\
\text { segmental PE }\end{array}$ & Fatal PE & Fatal PE & Fatal PE & Fatal PE & $\begin{array}{l}\text { Non-fatal Rt. } \\
\text { lobar PE }\end{array}$ \\
\hline $\begin{array}{l}\text { Cancer treatment } \\
\text { within } 4 \text { weeks at } \\
\text { the time of recurrent } \\
\text { VTE diagnosis }\end{array}$ & Chemotherapy & Chemotherapy & Chemotherapy & Chemotherapy & Chemotherapy & Chemotherapy & Chemotherapy \\
\hline Disease status & Stable disease & $\begin{array}{l}\text { Stable } \\
\text { disease }\end{array}$ & $\begin{array}{l}\text { Progressive } \\
\text { disease }\end{array}$ & $\begin{array}{l}\text { Stable } \\
\text { disease }\end{array}$ & $\begin{array}{l}\text { Progressive } \\
\text { disease }\end{array}$ & $\begin{array}{l}\text { Progressive } \\
\text { disease }\end{array}$ & $\begin{array}{l}\text { Progressive } \\
\text { disease }\end{array}$ \\
\hline
\end{tabular}

VTE, venous thromboembolism; Rt., right; PE, pulmonary embolism; DVT, deep vein thrombosis; Lt., left.

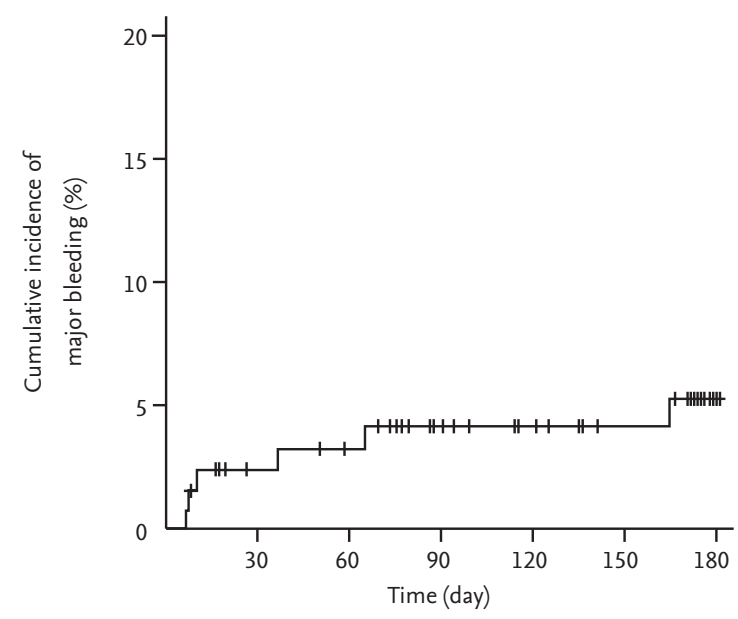

$\begin{array}{llllllll}\text { No. at risk } & 124 & 111 & 103 & 92 & 85 & 77 & 55\end{array}$

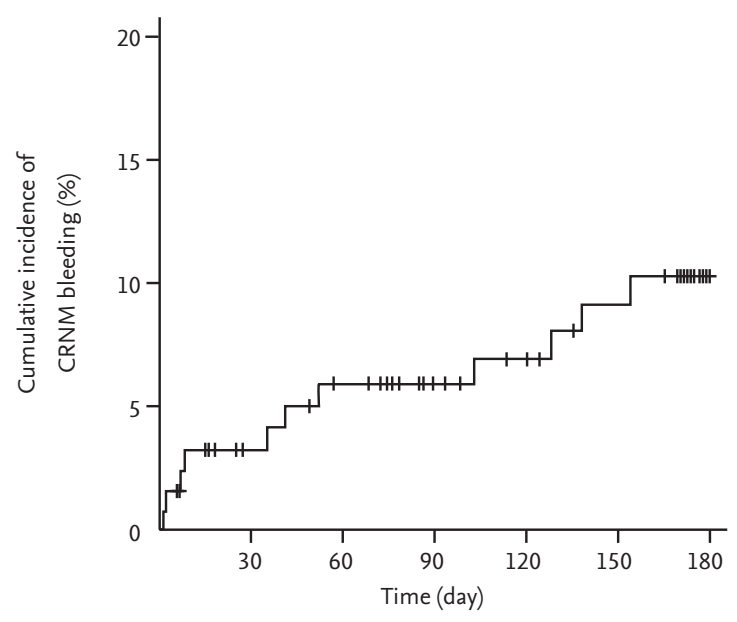

$\begin{array}{lllllllll}\text { B } & \text { No. at risk } & 124 & 108 & 98 & 87 & 80 & 72 & 50\end{array}$

Figure 4. Cumulative incidence of (A) major and (B) clinically relevant non-major (CRNM) bleeding events.

dental VTE events had similar rates of recurrent VTE and mortality in our study, as compared to those with symptomatic events, which was consistent with previous observations in cancer patients $[28,29]$. Thus, our study prospectively confirmed the impact of the incidental VTE on recurrent VTE and mortality in cancer-associated VTE.

The rate of major bleeding observed in this study was 


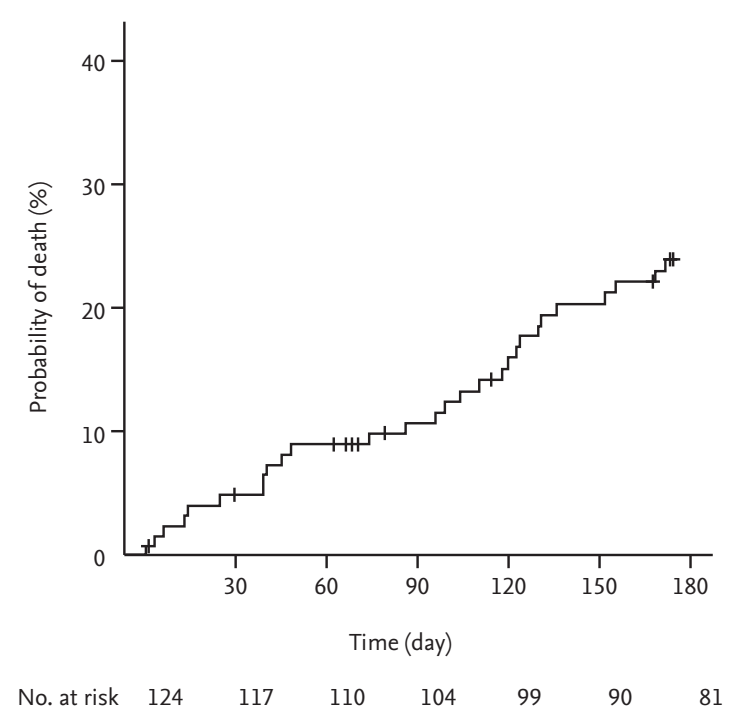

Figure 5. Overall mortality in the study population.

Table 3. Comparison of baseline characteristics, recurrent VTE, and bleeding events in the KVTE 1301 study with CLOT and CATCH trials

\begin{tabular}{|c|c|c|c|}
\hline Characteristic & $\begin{array}{c}\text { CLOT } \\
(\mathrm{n}=676)\end{array}$ & $\begin{array}{l}\text { CATCH } \\
(n=900)\end{array}$ & $\begin{array}{c}\text { KVTE 1301 } \\
(\mathrm{n}=124)\end{array}$ \\
\hline Asian population, \% & $\mathrm{O}$ & 44 & 100 \\
\hline Mean age, yr & 62 & 60 & 67 \\
\hline History of VTE, \% & 11.1 & 6.3 & 4.8 \\
\hline $\begin{array}{l}\text { ECOG performance } \\
\text { status } 2, \%\end{array}$ & $35 \cdot 5$ & 23.2 & 21.8 \\
\hline Metastatic disease, \% & $67 \cdot 3$ & $54 \cdot 7$ & $75 \cdot 0$ \\
\hline $\begin{array}{l}\text { Anti-cancer treat- } \\
\text { ment, \% }\end{array}$ & 77.7 & 52.9 & 88.7 \\
\hline $\begin{array}{l}\text { Cumulative 6-month } \\
\text { mortality, \% }\end{array}$ & $39 \cdot 3$ & 32.0 & 23.8 \\
\hline $\begin{array}{l}\text { Outcomes during } 6 \\
\text { months }\end{array}$ & $\begin{array}{l}\text { Dalteparin } \\
\quad(\mathrm{n}=338)\end{array}$ & $\begin{array}{l}\text { Tinzaparin } \\
(\mathrm{n}=449)\end{array}$ & $\begin{array}{c}\text { Rivarox- } \\
\text { aban } \\
(\mathrm{n}=124)\end{array}$ \\
\hline $\begin{array}{l}\text { Symptomatic recur- } \\
\text { rent VTE rate, \% }\end{array}$ & 9 & 7.2 & $5 \cdot 9$ \\
\hline Major bleeding, \% & 6 & 2.7 & $5 \cdot 3$ \\
\hline CRNM bleeding, \% & $14^{*}$ & 10.9 & 10.2 \\
\hline
\end{tabular}

VTE, venous thromboembolism; KVTE, Korean venous thromboembolism; CLOT, randomized Comparison of LMWH versus Oral anticoagulant therapy for the prevention of recurrent VTE in patients with Cancer; CATCH, Comparison of Acute Treatments in Cancer Haemostasis; ECOG, Eastern Cooperative Oncology Group; CRNM, clinically relevant non-major.

${ }^{a}$ Indicates the proportion of patients with any bleedings reported in the CLOT trial.
5.3\% for up to 6 months, slightly higher than anticipated. Although the CLOT trial reported major bleeding rate of $6 \%$ [2], the rate of major bleeding in the recent studies regarding DOACs was approximately $2 \%$ to $3 \%$ in cancer patients [14-17], which was similar to those found in LMWH group of the CATCH trial (2.7\%) [3]. This slightly higher than expected major bleeding rate could be partially explained by lower body weight or body mass index of our population, as compared with those in the CATCH trial [3] and the cancer subgroup of DOAC trials $[14,15,17]$. In addition, the fact that the majority of the study population was receiving anti-cancer treatments may raise the possibility that rivaroxaban activity is altered through interactions with chemotherapeutic agents and subsequently increases the risk of major bleeding. Although our study excluded patients treated with bevacizumab or other drugs with anti-angiogenic properties (i.e., sunitinib, sorafenib, imatinib, nilotinib, dasatinib, ramucirumab, etc.), we allowed the use of some chemotherapeutic or hormonal agents that potentially inhibit or induce P-glycoprotein transport or the $\mathrm{CYP}_{3} \mathrm{~A}_{4}$ pathway (i.e., doxorubicin, vinblastine, dexamethasone, tamoxifen, etc.) [8]. We could not analyze the impact of drug-drug interactions on bleeding events because only a small number of patients received these drugs. However, tamoxifen is known to increase the plasma level of rivaroxaban, but none of the patients treated with tamoxifen and rivaroxaban in this study experienced bleeding events (data not shown). Furthermore, the sum of both major and CRNM bleeding events (15.5\% for 6 months) was similar to those reported in the cancer subgroup of DOAC trials [14-17] and the CATCH trial [3]. These findings suggested that rivaroxaban did not universally increase the bleeding risk in cancer patients receiving active anti-cancer treatments. Thus, despite possible interactions between rivaroxaban and some anti-cancer drugs, we postulated that the impact of drug interactions on bleeding events may be minimal if agents with anti-angiogenic properties were not concomitantly used.

Interestingly, the gastrointestinal tract was the main site of major bleeding in our study population and onethird of major bleeding events were located at the site of unresected primary tumor, which was consistent with the results of meta-analysis in unselected populations [30] and cancer patients [31] with VTE and treated with 
DOACs. The mechanisms of gastrointestinal bleeding observed from rivaroxaban therapy may be explained by the local effect of unabsorbed anticoagulant in the lumen of the gastrointestinal tract and consequent potentiation in vulnerable lesions in combination with systemic drug effect [32]. Therefore, the use of DOACs may lead to increased risk of gastrointestinal bleeding in patients with vulnerable mucosal lesion on unresected primary tumors of the gastrointestinal tract and need to be avoided in this group of patients. However, because the use of any anticoagulants in these patients may be associated with increased risk of bleeding, it is difficult to determine the risk of gastrointestinal bleeding during the treatment with other anticoagulants (i.e., LMWH). Considering the theoretical absence of local anticoagulant effects of LMWH, it is necessary to evaluate the unresected primary gastrointestinal tumor as one of the factors for optimal selection of long-term anticoagulant treatment in patients with cancer-associated VTE.

Our study has several limitations. First, our study permitted the enrollment within 120 hours after confirmation of the qualifying VTE event or the start of anticoagulation therapy, which was longer than the CLOT (48 hours) and CATCH (72 hours) trials. Because the risk of recurrent VTE was highest during the first month of VTE episode [33], it is possible that some patients with recurrence at first week of qualifying VTE episode were not considered as candidates for this study, indicating potentials of selection bias. Our study enrolled patients with incidental thrombosis as well as with symptomatic VTE and the date of objective diagnosis in patients with incidental thrombosis was the date of radiologic studies for qualifying VTE episode. There is a time gap between radiologic studies and detection of thrombosis in asymptomatic patients in real clinical practice. Because of this, we allowed an additional 24- to 48-hour time period between objective diagnosis and study enrollment. However, despite this limitation, our study included the highest proportion of metastatic cancer patients with active anti-cancer treatments among studies ever conducted in this setting. Second, our findings may not be applicable to other ethnicities because of the single ethnicity (i.e., Korean) of our study participants. Third, although the optimal duration of rivaroxaban treatment in patients with cancer-associated VTE is be- yond the scope of this study, actual rates of recurrent VTE may have been underestimated owing to 6 months of treatment duration. There is a data showing that the risk of recurrent VTE is still clinically significant beyond 6 months of treatment [34]. In addition, edoxaban recently showed continued benefit of treatment beyond 6 months [35]. Therefore, rivaroxaban treatment should not be discontinued just because patients completed the 6-month period treatment. Fourth, our study contained a relatively small sample size with a wide range of CI because the goal of our study was to detect a promising, but not definitive benefit. Nevertheless, our results are sufficient to warrant assessment of rivaroxaban in a phase III trial because cancer-associated VTE patients consist a highly specific population with enormous unmet needs for treatment.

In conclusion, our results show that single targeted inhibition of coagulation system using rivaroxaban is effective and safe in patients with active cancer and symptomatic or incidental lower-extremity DVT, PE, or both. Further evaluation of our findings in phase III trials is under way.

\section{KEY MESSAGE}

1. Cumulative incidence of symptomatic recurrent venous thromboembolism (VTE) and major bleeding events were $5.9 \%$ and $5.3 \%$, respectively.

2. Oral administration of rivaroxaban offers a simple and convenient option to treat cancer-associated VTE

\section{Conflict of interest}

This study was supported by an independent research fund from Bayer Korea.

\section{Acknowledgments}

We would like to thank all participating patients and all study staff and nurses for their work in enrolling and managing patients. We would also like to acknowledge the contribution made by members of the independent central adjudication committee: Junshik Hong (Department of Internal Medicine, Seoul National University Hospital, Seoul, Korea) and Ji-Hyun Kwon (Department of Internal Medicine, Chungbuk National University 
Hospital, Cheongju, Korea), and sample size determination by Heeyoung Lee (Center for Preventive Medicine and Public Health, Seoul National University Bundang Hospital, Seoul, Korea).

\section{REFERENCES}

1. Young A, Chapman O, Connor C, Poole C, Rose P, Kakkar AK. Thrombosis and cancer. Nat Rev Clin Oncol 2012;9:437-449.

2. Lee AY, Levine MN, Baker RI, et al. Low-molecular-weight heparin versus a coumarin for the prevention of recurrent venous thromboembolism in patients with cancer. $\mathrm{N}$ Engl J Med 2003;349:146-153.

3. Lee AYY, Kamphuisen PW, Meyer G, et al. Tinzaparin vs warfarin for treatment of acute venous thromboembolism in patients with active cancer: a randomized clinical trial. JAMA 2015;314:677-686.

4. Kearon C, Akl EA, Ornelas J, et al. Antithrombotic therapy for VTE disease: CHEST guideline and expert panel report. Chest 2016;149:315-352.

5. Lyman GH, Khorana AA, Kuderer NM, et al. Venous thromboembolism prophylaxis and treatment in patients with cancer: American Society of Clinical Oncology clinical practice guideline update. J Clin Oncol 2013;31:21892204 .

6. Mandala M, Falanga A, Roila F; ESMO Guidelines Working Group. Management of venous thromboembolism (VTE) in cancer patients: ESMO Clinical Practice Guidelines. Ann Oncol 2011;22 Suppl 6:vi85-vig2.

7. Farge D, Bounameaux H, Brenner B, et al. International clinical practice guidelines including guidance for direct oral anticoagulants in the treatment and prophylaxis of venous thromboembolism in patients with cancer. Lancet Oncol 2016;17:e452-e466.

8. Lee AY, Peterson EA. Treatment of cancer-associated thrombosis. Blood 2013;122:2310-2317.

9. Schulman S, Kearon C, Kakkar AK, et al. Dabigatran versus warfarin in the treatment of acute venous thromboembolism. N Engl J Med 2009;361:2342-2352.

10. EINSTEIN-PE Investigators, Buller HR, Prins MH, et al. Oral rivaroxaban for the treatment of symptomatic pulmonary embolism. N Engl J Med 2012;366:1287-1297.

11. EINSTEIN Investigators, Bauersachs R, Berkowitz SD, et al. Oral rivaroxaban for symptomatic venous thrombo- embolism. N Engl J Med 2010;363:2499-2510.

12. Agnelli G, Buller HR, Cohen A, et al. Oral apixaban for the treatment of acute venous thromboembolism. N Engl J Med 2013;369:799-808.

13. Hokusai-VTE Investigators, Buller HR, Decousus H, et al. Edoxaban versus warfarin for the treatment of symptomatic venous thromboembolism. N Engl J Med 2013;369:1406-1415.

14. Agnelli G, Buller HR, Cohen A, et al. Oral apixaban for the treatment of venous thromboembolism in cancer patients: results from the AMPLIFY trial. J Thromb Haemost 2015;13:2187-2191.

15. Prins MH, Lensing AW, Brighton TA, et al. Oral rivaroxaban versus enoxaparin with vitamin $\mathrm{K}$ antagonist for the treatment of symptomatic venous thromboembolism in patients with cancer (EINSTEIN-DVT and EINSTEIN-PE): a pooled subgroup analysis of two randomized controlled trials. Lancet Haematol 2014;1:e37-e46.

16. Raskob GE, van Es N, Segers A, et al. Edoxaban for venous thromboembolism in patients with cancer: results from a non-inferiority subgroup analysis of the Hokusai-VTE randomised, double-blind, double-dummy trial. Lancet Haematol 2016;3:e379-e387.

17. Schulman S, Goldhaber SZ, Kearon C, et al. Treatment with dabigatran or warfarin in patients with venous thromboembolism and cancer. Thromb Haemost 2015;114:150-157.

18. Yhim HY, Bang SM. Direct oral anticoagulants in the treatment of cancer-associated venous thromboembolism. Blood Res 2014;49:77-79.

19. Piran S, Schulman S. Management of venous thromboembolism: an update. Thromb J 2016;14(Suppl 1):23.

20. Louzada ML, Carrier M, Lazo-Langner A, et al. Development of a clinical prediction rule for risk stratification of recurrent venous thromboembolism in patients with cancer-associated venous thromboembolism. Circulation 2012;126:448-454.

21. Schulman S, Kearon C; Subcommittee on Control of Anticoagulation of the Scientific and Standardization Committee of the International Society on Thrombosis and Haemostasis. Definition of major bleeding in clinical investigations of antihemostatic medicinal products in non-surgical patients. J Thromb Haemost 2005;3:692-694.

22. Ay C, Posch F, Kaider A, Zielinski C, Pabinger I. Estimating risk of venous thromboembolism in patients with cancer in the presence of competing mortality. J Thromb 
Haemost 2015;13:390-397.

23. van der Hulle T, den Exter PL, Kooiman J, van der Hoeven JJ, Huisman MV, Klok FA. Meta-analysis of the efficacy and safety of new oral anticoagulants in patients with cancer-associated acute venous thromboembolism. J Thromb Haemost 2014;12:1116-1120.

24. Jang MJ, Bang SM, Oh D. Incidence of venous thromboembolism in Korea: from the Health Insurance Review and Assessment Service database. J Thromb Haemost 2011;9:85-91.

25. Yhim HY, Jang MJ, Bang SM, et al. Incidence of venous thromboembolism following major surgery in Korea: from the Health Insurance Review and Assessment Service database. J Thromb Haemost 2014;12:1035-1043.

26. Lee KW, Bang SM, Kim S, et al. The incidence, risk factors and prognostic implications of venous thromboembolism in patients with gastric cancer. J Thromb Haemost 2010;8:540-547.

27. Yhim HY, Jang MJ, Kwak JY, et al. The incidence, risk factors, and prognosis of recurrent venous thromboembolism (VTE) in patients with advanced solid cancers receiving anticoagulation therapy after the diagnosis of index VTE. Thromb Res 2013;131:e133-e140.

28. den Exter PL, Hooijer J, Dekkers OM, Huisman MV. Risk of recurrent venous thromboembolism and mortality in patients with cancer incidentally diagnosed with pulmonary embolism: a comparison with symptomatic patients. J Clin Oncol 2011;29:2405-2409.
29. van der Hulle T, den Exter PL, Planquette B, et al. Risk of recurrent venous thromboembolism and major hemorrhage in cancer-associated incidental pulmonary embolism among treated and untreated patients: a pooled analysis of 926 patients. J Thromb Haemost 2016;14:105113.

30. Chai-Adisaksopha $\mathrm{C}$, Crowther $\mathrm{M}$, Isayama $\mathrm{T}$, Lim W. The impact of bleeding complications in patients receiving target-specific oral anticoagulants: a systematic review and meta-analysis. Blood 2014;124:2450-2458.

31. Schulman S, Shrum J, Majeed A. Management of bleeding complications in patients with cancer on DOACs. Thromb Res 2016;140 Suppl 1:S142-S147.

32. Desai J, Kolb JM, Weitz JI, Aisenberg J. Gastrointestinal bleeding with the new oral anticoagulants: defining the issues and the management strategies. Thromb Haemost 2013;110:205-212.

33. Kearon C, Hirsh J. Management of anticoagulation before and after elective surgery. N Engl J Med 1997;336:15061511.

34. Francis CW, Kessler CM, Goldhaber SZ, et al. Treatment of venous thromboembolism in cancer patients with dalteparin for up to 12 months: the DALTECAN Study. J Thromb Haemost 2015;13:1028-1035.

35. Raskob GE, van Es N, Verhamme P, et al. Edoxaban for the treatment of cancer-associated venous thromboembolism. N Engl J Med 2018;378:615-624. 